\title{
Solution du problème de point fixe de Schauder
}

\author{
par \\ Robert Cauty (Paris)
}

\begin{abstract}
We give an affirmative answer to Schauder's fixed point question.
\end{abstract}
1. Introduction. Le but de cet article est de prouver le théorème suivant, qui résout un problème connu de Schauder ([5], problème 54).

THÉORÈme. Soient $E$ un espace vectoriel topologique séparé, $C$ un sousensemble convexe de $E$ et $f$ une fonction continue de $C$ dans $C$. Si $f(C)$ est contenu dans un sous-ensemble compact de $C$, alors $f$ a un point fixe.

Pour la démonstration, nous utiliserons l'espace $P(X)$ des mesures probabilistes à support fini sur un compact $X$. Les éléments de $P(X)$ sont les combinaisons convexes de mesures de Dirac; pour simplifier les notations, nous écrivons une telle combinaison sous la forme $\sum_{i=1}^{n} \lambda_{i} x_{i}$ avec $x_{i} \in X$, $\lambda_{i} \geq 0$ et $\sum_{i=1}^{n} \lambda_{i}=1$. Soit $P_{n}(X)$ l'ensemble des mesures probabilistes sur $X$ dont le support a au plus $n$ éléments. $P_{n}(X)$ a une topologie compacte naturelle, et $P(X)=\bigcup_{n=1}^{\infty} P_{n}(X)$ est la limite inductive de la suite croissante de compacts $\left\{P_{n}(X)\right\}_{n=1}^{\infty}$. Nous identifions $X$ à $P_{1}(X)$. Le foncteur $P$ a la propriété importante suivante : pour tout compact $X$, toute fonction continue de $X$ dans un sous-ensemble convexe $C$ d'un e.v.t. peut se prolonger en une fonction continue (affine) de $P(X)$ dans $C$. Cela permet de réduire la démonstration du théorème à celle de la proposition suivante, qui en est un cas particulier.

Proposition. Pour tout compact $X$, toute fonction continue de $P(X)$ dans $X$ a un point fixe.

Pour voir que le théorème résulte de cette proposition, prenons un compact $X$ de $C$ contenant $f(C)$. L'inclusion de $X$ dans $C$ se prolonge en une fonction continue $r$ de $P(X)$ dans $C$. Alors, $g=f \circ r: P(X) \rightarrow X$ est

2000 Mathematics Subject Classification: 54H25, 47H10. 
continue, et il suffit de remarquer que tout point fixe de $g$ est aussi un point fixe de $f$.

Nous commencerons par montrer, en utilisant des résultats classiques de Shchepin sur les limites projectives, que si la proposition est vraie pour tout compact métrisable, alors elle est vraie pour tout compact. Nous montrerons ensuite que s'il existait un compact métrisable $X$ et une fonction continue sans point fixe $f$ de $P(X)$ dans $X$, alors il existerait un rétracte absolu $E$ et une fonction continue sans point fixe $h$ de $E$ dans $E$ d'image contenue dans un compact de $E$, en contradiction avec un résultat bien connu sur les points fixes. Pour obtenir $E$, nous construirons au lemme 3 un compact métrisable de dimension dénombrable $Z$ et une fonction continue $\varphi$ de $Z$ dans $X$ dont le prolongement naturel à $P(Z)$ a des propriétés particulières de relèvement approximatif. La possibilité de l'existence d'une telle fonction $\varphi$ a été suggérée par un résultat de Zarichnyi ([6], théorème 1$)$; malheureusement, la fonction de Zarichnyi ne possède pas toutes les propriétés dont nous avons besoin. La démonstration du lemme 3 est longue et occupe les paragraphes 4 et 5 ; nous conseillons au lecteur, avant de se plonger dans les détails de cette démonstration, de lire le début du paragraphe 5 où son idée géométrique est esquissée.

2. Réduction au cas métrisable. Dans ce paragraphe, nous prouverons que si la proposition est vraie pour tout compact métrisable, alors elle l'est pour tout compact.

Un système projectif d'espaces topologiques sur un ensemble ordonné filtrant $A$ sera noté $S=\left(X_{\alpha}, p_{\alpha}^{\beta}, A\right)$ (les $X_{\alpha}$ sont des espaces topologiques et, pour $\alpha \leq \beta, p_{\alpha}^{\beta}: X_{\beta} \rightarrow X_{\alpha}$ est continue). Nous notons $\lim S$ la limite projective de ce système, et $p_{\alpha}$ la projection de $\lim S$ dans $X_{\alpha}$. Si $B$ est un sous-ensemble filtrant de $A$, nous notons $S \mid B=\left(X_{\alpha}, p_{\alpha}^{\beta}, B\right)$ le système obtenu en restreignant l'ensemble des indices à $B$. Si $B$ est cofinal dans $A$, nous identifions naturellement $\lim (S \mid B)$ à $\lim S$. Si $S_{1}=\left(X_{\alpha}, p_{\alpha}^{\beta}, A\right)$ et $S_{2}=\left(Y_{\alpha}, q_{\alpha}^{\beta}, A\right)$ sont deux systèmes projectifs ayant le même ensemble d'indices, un morphisme de $S_{1}$ dans $S_{2}$ est une famille de fonctions continues $f_{\alpha}: X_{\alpha} \rightarrow Y_{\alpha}, \alpha \in A$, telle que $f_{\alpha} p_{\alpha}^{\beta}=q_{\alpha}^{\beta} f_{\beta}$ pour $\alpha \leq \beta$. Un tel morphisme induit une application continue $\lim \left(f_{\alpha}\right) \operatorname{de} \lim S_{1} \operatorname{dans} \lim S_{2}$.

Un système projectif $S=\left(X_{\alpha}, p_{\alpha}^{\beta}, A\right)$ est dit factorisable si, pour toute fonction continue $f: \lim S \rightarrow \mathbb{R}$, il existe un indice $\alpha \in A$ et une fonction continue $f_{\alpha}: X_{\alpha} \rightarrow \mathbb{R}$ tels que $f=f_{\alpha} p_{\alpha}$. Un système projectif $S=\left(X_{\alpha}, p_{\alpha}^{\beta}, A\right)$ est appelé un $\omega$-système projectif s'il vérifie les conditions suivantes :

(i) Tout sous-ensemble dénombrable totalement ordonné de $A$ a une borne supérieure. 
(ii) Pour tout sous-ensemble totalement ordonné $B$ de $A$ admettant une borne supérieure $\beta$, la fonction $\lim _{\alpha \in B} p_{\alpha}^{\beta}: X_{\beta} \rightarrow \lim (S \mid B)$ est un homéomorphisme.

(iii) Chaque $X_{\alpha}$ a une base dénombrable.

Un sous-ensemble $B$ d'un ensemble filtrant $A$ est dit fermé si, pour tout sous-ensemble totalement ordonné $C \subset B$ admettant une borne supérieure dans $A$, cette borne supérieure appartient à $B$.

Nous utiliserons le théorème spectral de Shchepin (voir [3], théorème 3.1.9) :

Lemme 1. Soient $S_{1}=\left(X_{\alpha}, p_{\alpha}^{\beta}, A\right)$ et $S_{2}=\left(Y_{\alpha}, q_{\alpha}^{\beta}, A\right)$ deux $\omega$-systèmes projectifs d'espaces complètement réguliers sur un même ensemble d'indices $A$, et soit $f$ une fonction continue de $\lim S_{1}$ dans $\lim S_{2}$. Si $S_{1}$ est factorisable et si, pour tout $\alpha \in A$, la projection $p_{\alpha}: \lim S_{1} \rightarrow X_{\alpha}$ est surjective, alors il existe un sous-ensemble cofinal fermé $B$ de $A$ et un morphisme de $S_{1} \mid B$ dans $S_{2} \mid B$ dont la limite est égale à $f$.

Notons que, puisque $B$ est cofinal et fermé, le système projectif $S_{1} \mid B$ fourni par ce lemme est lui aussi un $\omega$-système projectif factorisable.

Si $g: Y \rightarrow Z$ est une fonction continue, nous notons $P_{n}(g): P_{n}(Y) \rightarrow$ $P_{n}(Z)$ les fonctions induites par $g$.

Soient maintenant $X$ un compact non métrisable et $f: P(X) \rightarrow X$ une fonction continue. Il est connu que $X$ est la limite d'un $\omega$-système factorisable $S=\left(X_{\alpha}, p_{\alpha}^{\beta}, A\right)$ tel que les projections $p_{\alpha}: X \rightarrow X_{\alpha}$ soient surjectives (l'argument est, par exemple, celui de la proposition 3.2.17 de [3]). Par fonctorialité, nous obtenons les systèmes projectifs $P_{n}(S)=\left(P_{n}\left(X_{\alpha}\right), P_{n}\left(p_{\alpha}^{\beta}\right), A\right)$, $P_{1}(S)$ s'identifiant naturellement à $S$. Il est connu et élémentaire que les foncteurs $P_{n}$ commutent aux limites projectives de compacts. Puisque les $X_{\alpha}$ sont métrisables, les $P_{n}\left(X_{\alpha}\right)$ aussi. Il en résulte que $P_{n}(S)$ est un $\omega$ système projectif dont la limite s'identifie naturellement à $P_{n}(X)$. Puisque les projections $p_{\alpha}: X \rightarrow X_{n}$ sont surjectives, les $P_{n}\left(p_{\alpha}\right): P_{n}(X) \rightarrow P_{n}\left(X_{\alpha}\right)$ le sont aussi, donc $P_{n}(X)$ est factorisable ([3], corollaire 3.1.6). $P_{n}(S)$ et $S$ vérifient donc les hypothèses du lemme 1 . Compte tenu de la remarque faite après ce lemme, nous pouvons, partant de $A_{0}=A$, construire inductivement un sous-ensemble $A_{n}$ de $A_{n-1}$, cofinal et fermé dans $A_{n-1}$ (donc aussi dans $A$ ) et un morphisme $\left(f_{\alpha}^{n}\right)_{\alpha \in A_{n}}$ de $P_{n}(S) \mid A_{n}$ dans $S \mid A_{n}$ de limite $f \mid P_{n}(X)$. Alors, $B=\bigcap_{n=1}^{\infty} A_{n}$ est cofinal dans $A$ ([3], proposition 3.1.1).

Soit $\alpha \in B$. Pour $n<m$, nous avons $f_{\alpha}^{n} \circ P_{n}\left(p_{\alpha}\right)=p_{\alpha} \circ\left(f \mid P_{n}(X)\right)$ et $f_{\alpha}^{m} \circ P_{m}\left(p_{\alpha}\right)=p_{\alpha} \circ\left(f \mid P_{m}(X)\right)$. Puisque $P_{n}\left(p_{\alpha}\right)=P_{m}\left(p_{\alpha}\right) \mid P_{n}(X)$ est surjective, ces égalités entraînent que $f_{\alpha}^{n}=f_{\alpha}^{m} \mid P_{n}\left(X_{\alpha}\right)$. Nous pouvons donc définir une fonction continue $f_{\alpha}$ de $P\left(X_{\alpha}\right)$ dans $X_{\alpha}$ en posant $f_{\alpha} \mid P_{n}\left(X_{\alpha}\right)=$ $f_{\alpha}^{n}$ pour tout $n \geq 1$. Si la proposition est vraie pour les compacts métrisables, alors l'ensemble $F_{\alpha}$ des points fixes de $f_{\alpha}$ est un sous-ensemble compact 
non vide de $X_{\alpha}$. Si $\alpha \leq \beta$ appartiennent à $B$, alors $p_{\alpha}^{\beta}\left(F_{\beta}\right) \subset F_{\alpha}$, et nous obtenons un système projectif de compacts $S_{F}=\left(F_{\alpha}, p_{\alpha}^{\beta} \mid F_{\beta}, B\right)$ dont la limite est un sous-ensemble non vide $\operatorname{de} \lim (S \mid B)=X$, et chaque point de $\lim S_{F}$ est un point fixe de la limite $f \mid X$ du morphisme $\left(f_{\alpha}^{1}\right)_{\alpha \in B}$.

3. Le cas métrisable. Pour un compact métrisable $X$, nous notons $E(X)$ l'espace vectoriel topologique libre engendré par $X$ et $\mathcal{T}(X)$ l'ensemble des topologies d'espace métrique linéaire sur $E(X)$ qui sont moins fines que la topologie libre. Une description détaillée de $E(X)$ est donnée dans [1]; en se reportant à cette description, on constate immédiatement que $P(X)$ est homéomorphe à un sous-ensemble convexe fermé de $E(X)$. Le lemme suivant est un cas particulier du lemme 2.2 de [1].

Lemme 2. Soient $X$ un compact métrisable et $f$ une fonction continue de $P(X)$ dans un espace métrisable $M$. Alors il existe $\tau \in \mathcal{T}(X)$ telle que $f:(P(X), \tau) \rightarrow M$ soit continue.

Si $\mathcal{U}$ est une famille de sous-ensembles d'un espace $X$ et $A$ un sousensemble de $X$, nous posons $\operatorname{St}(A, \mathcal{U})=\bigcup\{U \in \mathcal{U} \mid A \cap U \neq \emptyset\}$, et nous notons $\operatorname{St}(\mathcal{U})=\{\operatorname{St}(U, \mathcal{U}) \mid U \in \mathcal{U}\}$. Deux fonctions $f, g: Y \rightarrow X$ sont $\mathcal{U}$-proches si, pour tout $y \in Y$, il existe un élément de $\mathcal{U}$ contenant $f(y)$ et $g(y)$. L'espace $X$ est de dimension dénombrable s'il est réunion dénombrable de sous-espaces de dimension zéro.

Dans le lemme suivant, $\widehat{\varphi}: P(Z) \rightarrow P(X)$ est le prolongement naturel de $\varphi$.

Lemme 3. Soit $X$ un compact métrisable. Il existe un compact métrisable $Z$ et une fonction continue $\varphi$ de $Z$ dans $X$ ayant les propriétés suivantes :

(i) $Z$ est de dimension dénombrable.

(ii) Si $\tau \in \mathcal{T}(X)$ et $\tau^{\prime} \in \mathcal{T}(Z)$ sont telles que $\widehat{\varphi}:\left(P(Z), \tau^{\prime}\right) \rightarrow(P(X), \tau)$ soit continue, alors, pour tout recouvrement $\tau$-ouvert $\mathcal{U}$ de $P(X)$, tout complexe simplicial dénombrable localement fini $N$ et toute fonction continue $\xi: N \rightarrow X$, il existe une fonction continue $\eta: N \rightarrow\left(P(Z), \tau^{\prime}\right)$ telle que $\widehat{\varphi} \circ \eta$ soit $\mathcal{U}$-proche de $\xi$ et que $\eta(N) \cup P_{2}(Z)$ soit $\tau^{\prime}$-compact.

Ce lemme sera prouvé au paragraphe 5 .

Supposons qu'il existe un compact métrisable $X$ et une fonction continue sans point fixe $f$ de $P(X)$ dans $X$. Soit $\varphi: Z \rightarrow X$ la fonction du lemme 3 . Le lemme 2 nous permet de trouver $\tau \in \mathcal{T}(X)$ telle que $f:(P(X), \tau) \rightarrow X$ soit continue, puis $\tau^{\prime} \in \mathcal{T}(Z)$ telle que $\widehat{\varphi}:\left(P(Z), \tau^{\prime}\right) \rightarrow(P(X), \tau)$ soit continue. Puisque $f$ est sans point fixe, nous pouvons trouver un recouvrement $\tau$ ouvert $\mathcal{U}$ de $P(X)$ vérifiant

$$
U \cap f(U)=\emptyset \quad \text { pour tout } U \in \mathcal{U} .
$$


Soit $\mathcal{V}$ un recouvrement $\tau$-ouvert de $P(X)$ tel que $\operatorname{St}(\mathcal{V})$ soit plus fin que $\mathcal{U}$. Pour tout $n \geq 1$, tout point de $P_{n+1}(Z) \backslash P_{n}(Z)$ a, dans $P_{n+1}(Z)$, un voisinage homéomorphe à un sous-ensemble de $Z^{n+1} \times \Delta_{n}$, où $\Delta_{n}$ est un $n$-simplexe; ce voisinage est donc de dimension dénombrable. Puisque les $P_{n}(Z)$ ont des bases dénombrables, il en résulte qu'ils sont de dimension dénombrable, donc il en est de même de $\left(P(Z), \tau^{\prime}\right)$. D'après [4], $\left(P(Z), \tau^{\prime}\right)$ est un rétracte absolu; comme cet espace est séparable, nous pouvons trouver un complexe simplicial dénombrable localement fini $N$ et des fonctions continues $\mu:\left(P(Z), \tau^{\prime}\right) \rightarrow N$ et $\xi: N \rightarrow\left(P(Z), \tau^{\prime}\right)$ vérifiant

$$
\xi \circ \mu \text { est } \widehat{\varphi}^{-1}(\mathcal{V}) \text {-proche de } \operatorname{id}_{P(Z)} \text {. }
$$

La fonction $f \circ \widehat{\varphi} \circ \xi: N \rightarrow X$ est continue. D'après (ii) du lemme 3, nous pouvons trouver une fonction continue $\eta: N \rightarrow\left(P(Z), \tau^{\prime}\right)$ vérifiant

$$
\begin{gathered}
\widehat{\varphi} \circ \eta \text { est } \mathcal{V} \text {-proche de } f \circ \widehat{\varphi} \circ \xi, \\
\eta(N) \cup P_{2}(Z) \text { est } \tau^{\prime} \text {-compact. }
\end{gathered}
$$

Alors, $h=\eta \circ \mu$ est une fonction continue de $\left(P(Z), \tau^{\prime}\right)$ dans lui-même dont l'image est contenue dans le sous-ensemble $\tau^{\prime}$-compact $\eta(N) \cup P_{2}(Z)$. D'après une propriété de point fixe bien connue des rétractes absolus ([2], théorème II.10.8), $h$ a un point fixe $x_{0}$. D'après (3), il y a un élément $V_{1}$ de $\mathcal{V}$ contenant $\widehat{\varphi}\left(x_{0}\right)=\widehat{\varphi} \circ \eta \circ \mu\left(x_{0}\right)$ et $f \circ \widehat{\varphi} \circ \xi \circ \mu\left(x_{0}\right)$. D'après $(2)$, il y a un élément $V_{2}$ de $\mathcal{V}$ contenant $\widehat{\varphi}\left(x_{0}\right)$ et $\widehat{\varphi} \circ \xi \circ \mu\left(x_{0}\right)$. Alors $\widehat{\varphi}\left(x_{0}\right) \in V_{1} \cap V_{2}$ et, puisque $\operatorname{St}(\mathcal{V})$ est plus fin que $\mathcal{U}$, il y a un élément $U_{0}$ de $\mathcal{U}$ contenant $V_{1} \cup V_{2}$, donc les deux points $\widehat{\varphi} \circ \xi \circ \mu\left(x_{0}\right)$ et $f \circ \widehat{\varphi} \circ \xi \circ \mu\left(x_{0}\right)$, en contradiction avec (1).

4. Quelques constructions auxiliaires. Dans les deux derniers paragraphes, nous aurons besoin d'utiliser plusieurs subdivisions d'un même complexe. Nous y ferons donc, contrairement à ce que nous avons fait au paragraphe précédent, une distinction formelle entre un complexe simplicial abstrait $K$ et sa réalisation géométrique $|K|$. Pour tout simplexe $\sigma$ de $K$, nous notons $\partial \sigma$ son bord, $\stackrel{\circ}{\sigma}=|\sigma| \backslash|\partial \sigma|$ et $b_{\sigma}$ son barycentre. Si $\sigma, \tau$ sont deux simplexes de $K$, la notation $\sigma \leq \tau$ signifie que $\sigma$ est face de $\tau$. Si $v$ est un sommet de $K$, nous notons $\overline{\operatorname{St}}(v, K)$ le sous-complexe de $K$ formé de tous les simplexes contenant $v$ et de leurs faces, et $\operatorname{St}(v, K)$ l'intérieur de $|\overline{\operatorname{St}}(v, K)|$ dans $|K|$. Nous notons de la même façon une application simpliciale de $K$ dans $L$ et la fonction continue de $|K|$ dans $|L|$ qu'elle engendre. Nous notons $K^{(n)}$ la $n^{\text {ième }}$ subdivision barycentrique de $K\left(K^{(0)}=K\right)$; les simplexes de $K^{(1)}$ sont les suites $\left(b_{\sigma_{0}}, \ldots, b_{\sigma_{k}}\right)$ où $\sigma_{0}, \ldots, \sigma_{k}$ sont des simplexes de $K$ vérifiant $\sigma_{0} \leq \ldots \leq \sigma_{k}$. Si $f: K \rightarrow L$ est une application simpliciale, nous définissons par récurrence une application simpliciale $f^{(n)}: K^{(n)} \rightarrow L^{(n)}$ comme suit $:$ si $\sigma \in K$ et si $f(\sigma)=\tau$, alors $f^{(1)}\left(b_{\sigma}\right)=b_{\tau}$, 
et si $f^{(n-1)}$ est définie, alors $f^{(n)}=\left(f^{(n-1)}\right)^{(1)}$. Si $K^{\prime}$ est une subdivision de $K$, nous identifions naturellement $\left|K^{\prime}\right|$ et $|K|$; en particulier, nous écrirons systématiquement $|K|$ au lieu de $\left|K^{(n)}\right|$.

Soit $K$ un complexe simplicial fini de dimension $\leq n$. Construisons un complexe simplicial $M(K, n)$ comme suit : les sommets de $M(K, n)$ sont les couples $\left(b_{\sigma}, m\right)$ où $\sigma$ est un simplexe de $K$ et $m$ un entier vérifiant $\operatorname{dim} \sigma \leq m \leq n$. Un ensemble fini $\left\{\left(b_{\sigma_{0}}, m_{0}\right), \ldots,\left(b_{\sigma_{k}}, m_{k}\right)\right\}$ de tels couples est un simplexe si la numérotation peut être choisie de façon que $\sigma_{0} \leq$ $\ldots \leq \sigma_{k}$ et $m_{0}<\ldots<m_{k}$. Nous définissons une application simpliciale $r=r(K, n)$ de $M(K, n)$ sur $K^{(1)}$ en posant $r\left(\left(b_{\sigma}, m\right)\right)=b_{\sigma}$. Évidemment, le sous-complexe plein de $M(K, n)$ déterminé par les sommets de la forme $\left(b_{\sigma}, \operatorname{dim} \sigma\right)$ est isomorphe à $K^{(1)}$; nous identifions $K^{(1)}$ à ce sous-complexe, ce qui nous permet de regarder $r$ comme une rétraction simpliciale de $M(K, n) \operatorname{sur} K^{(1)}$.

Lemme 4. Il existe une homotopie $H:|M(K, n)| \times[0,1] \rightarrow|M(K, n)|$ vérifiant

(i) $r \circ H(x, t)=r(x)$ quels que soient $x$ et $t$,

(ii) $H(x, t)=x$ si $t=0$ ou si $x \in|K|$,

(iii) $H(x, 1)=r(x)$ pour tout $x$.

Démonstration. Posons $H(x, 0)=x$. Pour un $i \in\{0, \ldots, n-1\}$, supposons $H \mid(|M(K, n)| \times[0, i / n])$ construite de façon que, pour tout $x, H(x, i / n)$ appartienne à $\left|L_{i}\right|$, où $L_{i}$ est le sous-complexe plein de $M(K, n)$ engendré par les sommets $\left(b_{\sigma}, m\right)$ tels que $m=\operatorname{dim} \sigma$ ou $m \geq i+1$. Pour $i / n \leq$ $t \leq(i+1) / n$ et $v=\left(b_{\sigma}, m\right)$ un sommet de $L_{i}$, posons $G_{i}(v, t)=v$ si $m \neq i+1$ et $G_{i}(v, t)=(i+1-n t)\left(b_{\sigma}, m\right)+(n t-i)\left(b_{\sigma}, \operatorname{dim} \sigma\right)$ si $m=i+1$. Si $\left(b_{\sigma_{0}}, m_{0}\right), \ldots,\left(b_{\sigma_{k}}, m_{k}\right)$, où $m_{0}<\ldots<m_{k}$, sont les sommets d'un simplexe de $L_{i}$, et s'il existe un indice $j$ tel que $m_{j}=i+1 \neq \operatorname{dim} \sigma_{j}$ alors les points $\left(b_{\sigma_{0}}, m_{0}\right), \ldots,\left(b_{\sigma_{j-1}}, m_{j-1}\right),\left(b_{\sigma_{j}}, \operatorname{dim} \sigma_{j}\right),\left(b_{\sigma_{j}}, m_{j}\right), \ldots,\left(b_{\sigma_{k}}, m_{k}\right)$ sont les sommets d'un simplexe de $L_{i}$ (si $j>0$, alors $m_{j-1}<i+1$, donc $\left.m_{j-1}=\operatorname{dim} \sigma_{j-1} \leq \operatorname{dim} \sigma_{j}\right)$. Nous pouvons donc prolonger $G_{i}$ à $\left|L_{i}\right| \times[i / n,(i+1) / n]$ de façon que, pour tout simplexe $\tau$ de $L_{i}$ et tout $t \in[i / n,(i+1) / n]$, la restriction de $G_{i}$ à $|\tau| \times\{t\}$ soit affine. Pour $i / n \leq$ $t \leq(i+1) / n$, posons $H(x, t)=G_{i}(H(x, i / n), t)$. Alors, $H(x,(i+1) / n)$ appartient à $\left|L_{i+1}\right|$. Puisque $G \mid\left(\left|L_{i}\right| \times\{t\}\right)$ est affine, la condition (i) résulte de ce que $r \circ G_{i}(v, t)=r(v)$ pour tout sommet $v$, (ii) est évidente, et (iii) résulte du fait que $L_{n}=K^{(1)}$.

Pour $k \leq n$, soit $M^{k}(K, n)$ le sous-complexe plein de $M(K, n)$ engendré par les sommets $\left(b_{\sigma}, m\right)$ tels que $m \leq k$. Pour $x \in|M(K, n)|$, soit $\nu(K, n)(x)=\nu(x)$ la somme des coordonnées barycentriques de $x$ sur les sommets de $M(K, n) \backslash K^{(1)}$. 
Lemme 5. Pour tout $\varepsilon>0$, il existe une fonction continue $h_{\varepsilon}$ de $|M(K, n)|$ dans $|M(K, n)|$ vérifiant

(i) $r \circ h_{\varepsilon}=r$,

(ii) $h_{\varepsilon}|| K \mid=\mathrm{id}_{|K|}$,

(iii) $h_{\varepsilon}^{-1}\left(\left|M^{k}(K, n)\right|\right)=\left|M^{k}(K, n)\right|$ pour tout $k \leq n$,

(iv) $\nu\left(h_{\varepsilon}(x)\right) \leq \varepsilon$ pour tout $x \in|M(K, n)|$.

Démonstration. L'homotopie $H$ du lemme 4 a la propriété que, pour tout $x \in|M(K, n)|$, la fonction $\nu(H(x, \cdot))$ est décroissante. Par suite, les fonctions $\alpha(x)=\sup \{t \in[0,1] \mid \nu(H(x, t)) \geq \varepsilon\}(\sup \emptyset=0)$ et $\beta(x)=\inf \{t \in[0,1] \mid$ $\nu(H(x, t)) \leq \varepsilon / 2\}$ sont respectivement semi-continues supérieurement et inférieurement. Puisque $\alpha(x) \leq \beta(x)$ pour tout $x$, nous pouvons trouver une fonction continue $\gamma:|M(K, n)| \rightarrow[0,1]$ telle que $\alpha(x) \leq \gamma(x) \leq \beta(x)$ pour tout $x$. Posons $h_{\varepsilon}(x)=H(x, \gamma(x))$. La condition (iv) est vérifiée par définition de $\gamma$, et (i) (resp. (ii)) résulte de (i) (resp. (ii)) du lemme 4.

Soit $x$ un point de $|M(K, n)|$, et soient $\left(b_{\sigma_{0}}, m_{0}\right), \ldots,\left(b_{\sigma_{j}}, m_{j}\right)$ les sommets de $M(K, n)$ sur lesquels les coordonnées barycentriques de $x$ sont non nulles, numérotés de façon que $m_{0}<\ldots<m_{j}$. Si $x \in\left|M^{k}(K, n)\right|$, alors $m_{j} \leq$ $k$, et $H(x, \gamma(x))$ a, par construction de $H$, une coordonnée nulle sur tout sommet $\left(b_{\sigma}, m\right)$ tel que $m>m_{j}$, donc $h(x) \in\left|M^{k}(K, n)\right|$. Si $x \notin\left|M^{k}(K, n)\right|$, alors $m_{j}>k$, et nous avons $H(x, t)=H\left(x, m_{j} / n\right) \in|K|$ pour $t \geq m_{j} / n$, donc $\gamma(x) \leq \beta(x)<m_{j} / n$, et $H(x, \gamma(x))$ a une coordonnée barycentrique non nulle sur $\left(b_{\sigma_{j}}, m_{j}\right)$, ce qui montre que $h_{\varepsilon}(x) \notin\left|M^{k}(K, n)\right|$, d'où (iii).

Dans le lemme suivant, $d$ est une distance continue sur $|K|$.

Lemme 6. Pour tout entier $l \geq 1$ et tout $\varepsilon>0$, il existe une fonction continue $\zeta$ de $\left|M\left(K^{(l)}, n\right)\right|$ dans $|M(K, n)|$ vérifiant

(i) $d\left(r(K, n) \circ \zeta(x), r\left(K^{(l)}, n\right)(x)\right)<\varepsilon$ pour tout $x \in\left|M\left(K^{(l)}, n\right)\right|$,

(ii) $\zeta|| K \mid=\operatorname{id}_{|K|}$,

(iii) $\zeta^{-1}\left(\left|M^{k}(K, n)\right|\right) \subset\left|M^{k}\left(K^{(l)}, n\right)\right|$ pour tout $k \leq n$,

(iv) pour tout simplexe $\sigma$ de $K^{(1)}, \zeta\left(r\left(K^{(l)}, n\right)^{-1}(|\sigma|)\right) \subset r(K, n)^{-1}(|\sigma|)$,

(v) $\nu(\zeta(x)) \leq \varepsilon$ pour tout $x \in\left|M\left(K^{(l)}, n\right)\right|$.

Démonstration. Il suffit de construire $\zeta$ de façon qu'elle vérifie les conditions (i)-(iv), car alors, si $h_{\varepsilon}$ est la fonction du lemme $5, h_{\varepsilon} \circ \zeta$ vérifiera toutes les conditions $(\mathrm{i})-(\mathrm{v})$. Nous pouvons supposer $l=1$, le cas général s'obtenant en composant $l$ telles fonctions. Nous prouverons, par récurrence sur la dimension de $K$, l'existence de fonctions $\zeta$ vérifiant les conditions (i)-(iv) ainsi que la suivante :

(vi) Si $x \in\left|M^{k}\left(K^{(1)}, n\right)\right|$ et si $\zeta(x)$ a une coordonnée barycentrique non nulle sur le sommet $\left(b_{\sigma}, m\right)$ de $M(K, n)$, alors ou bien $m \leq k$, ou bien $m=\operatorname{dim} \sigma$. 
Si $\operatorname{dim} K=0$, les complexes $M(K, n)$ et $M\left(K^{(1)}, n\right)$ sont égaux et $\zeta=$ identité convient. Supposons $\operatorname{dim} K=d>0$ et le lemme vrai pour les complexes de dimension $\leq d-1$. Pour simplifier les notations, posons $r=$ $r(K, n)$ et $r_{1}=r\left(K^{(1)}, n\right)$. Soit $K_{d-1}$ le $(d-1)$-squelette de $K$, et soit $\zeta^{\prime}$ : $r_{1}^{-1}\left(\left|K_{d-1}\right|\right)=\left|M\left(K_{d-1}^{(1)}, n\right)\right| \rightarrow\left|M\left(K_{d-1}, n\right)\right|=r^{-1}\left(\left|K_{d-1}\right|\right)$ une fonction vérifiant les conditions (i)-(iv) et (vi) pour un certain $\varepsilon^{\prime} \leq \varepsilon$. Soit $s$ un $d$ simplexe de $K$. Soit $V$ l'ensemble des sommets de $r_{1}^{-1}(|s|)$, et soit $S$ (resp. $T$ ) l'ensemble des $v \in V$ tels que $r_{1}(v)$ appartienne à $\operatorname{St}\left(b_{s}, K^{(2)}\right)$ (resp. $\left.\partial s\right)$; $V$ est la réunion disjointe de $S$ et $T$. Pour $v \in V$ et $x \in r_{1}^{-1}(|s|)$, nous notons $\lambda_{v}(x)$ la coordonnée barycentrique de $x$ sur $v$, et nous posons $\alpha(x)=$ $\sum_{v \in S} \lambda_{v}(x)$ et $\beta(x)=\sum_{v \in T} \lambda_{v}(x)=1-\alpha(x)$. Pour $v=\left(b_{\sigma}, m\right) \in S$, soit $\theta(v)=\left(b_{s}, \max (m, d)\right)$. Définissons une fonction continue $\xi: r_{1}^{-1}(\stackrel{\circ}{s}) \rightarrow$ $r^{-1}\left(b_{s}\right)$ par

$$
\xi(x)=\frac{1}{\alpha(x)} \sum_{v \in S} \lambda_{v}(x) \theta(v),
$$

et une fonction continue $\eta: r_{1}^{-1}\left(|s| \backslash\left|\overline{\mathrm{St}}\left(b_{s}, K^{(2)}\right)\right|\right) \rightarrow r_{1}^{-1}(|\partial s|)$ par

$$
\eta(x)=\frac{1}{\beta(x)} \sum_{v \in T} \lambda_{v}(x) v .
$$

Soit $\varrho:|s| \backslash\left\{b_{s}\right\} \rightarrow|\partial s|$ la projection radiale, et soit $\pi=\varrho \circ r_{1}:$ $r_{1}^{-1}\left(|s| \backslash\left\{b_{s}\right\}\right) \rightarrow|\partial s|$. Pour tout $x \in r_{1}^{-1}\left(|s| \backslash\left\{b_{s}\right\}\right)$, soit $\mu(x) \in[0,1]$ le nombre tel que $r_{1}(x)=(1-\mu(x)) \pi(x)+\mu(x) b_{s}$. Les fonctions $\pi$ et $\mu$ sont continues sur $r_{1}^{-1}\left(|s| \backslash\left\{b_{s}\right\}\right)$. Prenons des voisinages ouverts $U_{1}$ et $U_{2}$ de $r_{1}^{-1}(|\partial s|) \backslash|s|$ dans $r_{1}^{-1}(|s|) \backslash|s|$ vérifiant $\bar{U}_{2} \subset r_{1}^{-1}\left(|s| \backslash\left|\overline{\operatorname{St}}\left(b_{s}, K^{(2)}\right)\right|\right)$, $\bar{U}_{2} \cap|s|=|\partial s|$ et $\bar{U}_{1} \subset U_{2} \cup|\partial s|$, puis des fonctions continues $\gamma$ et $\delta$ de $r_{1}^{-1}(|s|) \backslash|s|$ dans $[0,1]$ telles que $\gamma(x)=0$ si $x \in r_{1}^{-1}(|\partial s|) \backslash|s|, \gamma(x)=1$ si $x \notin U_{1}, \delta(x)=0$ si $x \in \bar{U}_{1}$ et $\delta(x)=1$ si $x \notin U_{2}$. $H$ étant l'homotopie construite au lemme 4 , définissons $\zeta \mid r_{1}^{-1}(|s|): r_{1}^{-1}(|s|) \rightarrow r^{-1}(|s|)$ par

$$
\zeta(x)= \begin{cases}\zeta^{\prime}(x) & \text { si } x \in r_{1}^{-1}(|\partial s|), \\ (1-\mu(x)) H\left(\zeta^{\prime} \circ \eta(x), \gamma(x)\right)+\mu(x) \xi(x) & \text { si } x \in \bar{U}_{1} \backslash r_{1}^{-1}(|\partial s|), \\ (1-\mu(x))\left[(1-\delta(x)) r \circ \zeta^{\prime} \circ \eta(x)+\delta(x) \pi(x)\right]+\mu(x) \xi(x) & \text { si } x \in \bar{U}_{2} \backslash\left(U_{1} \cup|s|\right), \\ (1-\mu(x)) \pi(x)+\mu(x) \xi(x) & \text { si } x \in r_{1}^{-1}\left(\stackrel{s}{s} \backslash\left\{b_{s}\right\}\right) \backslash U_{2}, \\ \xi(x) & \text { si } x \in r_{1}^{-1}\left(\left\{b_{s}\right\}\right) .\end{cases}
$$

Montrons que cette définition a un sens. Pour cela, notons d'abord que le choix des fonctions $\gamma$ et $\delta$ et les conditions (ii) et (iii) du lemme 4 garantissent la compatibilité des fonctions définissant $\zeta$. Il reste donc à vérifier que les points dont on prend des combinaisons convexes pour définir $\zeta$ sont dans un même simplexe de $|M(K, n)|$. Soit $x \in r_{1}^{-1}\left(\stackrel{\circ}{s} \backslash\left\{b_{s}\right\}\right)$, et soit $|\tau|$ un simplexe de $\left|(\partial s)^{(1)}\right|$ contenant $\pi(x)$. En ajoutant aux sommets de $\tau$ ceux 
de la forme $\left(b_{s}, m\right), d \leq m \leq n$, nous obtenons un simplexe $|\widehat{\tau}|$ de $|M(K, n)|$ contenant les deux points $\xi(x)$ et $\pi(x)$. Si $x \in r_{1}^{-1}\left(\stackrel{\circ}{s} \backslash\left|\overline{\operatorname{St}}\left(b_{s}, K^{(2)}\right)\right|\right)$, soient $\left(b_{\sigma_{0}}, m_{0}\right), \ldots,\left(b_{\sigma_{p}}, m_{p}\right)$ les éléments de $V$ sur lesquels les coordonnées barycentriques de $x$ sont $>0$, rangés de façon que $\sigma_{0} \leq \ldots \leq \sigma_{p}$ et $m_{0}<\ldots<m_{p}$. Il existe $q \in\{0, \ldots, p-1\}$ tel que $\sigma_{i} \in(\partial s)^{(1)}$ si, et seulement si, $i \leq q$, et $\left|\sigma_{q}\right|$ est contenu dans $|\tau|$. Puisque $\zeta^{\prime}$ vérifie (iv), $r \circ \zeta^{\prime} \circ \eta(x)$ appartient à $|\tau| \subset|\widehat{\tau}|$. Si $x \in U_{1}$, soit $\left|\sigma^{\prime}\right|$ le plus petit simplexe de $|M(K, n)|$ contenant $H\left(\zeta^{\prime} \circ \eta(x), \gamma(x)\right)$. En utilisant la définition de $\eta$, les propriétés (iv) et (vi) (vérifiées par $\zeta^{\prime}$ ), la condition (i) du lemme 4 et le fait que, par construction, $H\left(\left|M^{k}(K, n)\right| \times[0,1]\right) \subset\left|M^{k}(K, n)\right|$ pour tout $k$, on constate que si $\left(b_{\sigma}, m\right)$ est un sommet de $\sigma^{\prime}$, alors $|\sigma| \subset|\partial s|$ et $m \leq \max \left(\operatorname{dim} \sigma, m_{q}\right)<\max \left(d, m_{q+1}\right)$, donc, en ajoutant aux sommets de $\sigma^{\prime}$ ceux de la forme $\left(b_{s}, m^{\prime}\right)$ avec $\max \left(d, m_{q+1}\right) \leq m^{\prime} \leq n$, nous obtenons un simplexe de $|M(K, n)|$ contenant à la fois $H\left(\zeta^{\prime} \circ \eta(x), \gamma(x)\right)$ et $\xi(x)$.

La continuité de $\zeta$ en un point de $r_{1}^{-1}\left(\stackrel{s}{s} \backslash\left\{b_{s}\right\}\right)$ résulte de la continuité des fonctions utilisées pour définir $\zeta$ sur leurs domaines de définition. La continuité de $\zeta$ en un point de $r_{1}^{-1}\left(b_{s}\right)$ (resp. $\left.r_{1}^{-1}(|\partial s|) \backslash|\partial s|\right)$ résulte du fait que si $\left\{y_{i}\right\}_{i=1}^{\infty}$ est une suite de points de $r^{-1}\left(b_{s}\right)$ (resp. $\left.r^{-1}(|\partial s|)\right)$ convergeant vers un point $y_{0}$, alors la suite $\left\{\left(1-t_{i}\right) y_{i}+t_{i} z_{i}\right\}$ converge vers $y_{0}$ quels que soient les $z_{i}$ si $\left\{t_{i}\right\}$ tend vers zéro. Soit $x \in|\partial s|$. Pour tout voisinage $W$ de $x$ dans $r^{-1}(|\partial s|)$ et pour $0<e<1$, soit $O(W, e)$ l'ensemble des points de $r^{-1}(|s|)$ de la forme $(1-t) y+t z$ où $y \in W, 0 \leq t<e$ et $z$ est n'importe quel élément de $r^{-1}(|s|)$ pour lequel la combinaison convexe est définie. Les ensembles $O(W, e)$ forment une base de voisinages de $x$ dans $r^{-1}(|s|)$. Il est alors clair que, pour vérifier la continuité de $\zeta$ en $x$, il suffit de trouver un voisinage $P$ de $x$ dans $r_{1}^{-1}(|s|)$ tel que $H\left(\zeta^{\prime} \circ \eta(z), u\right)$ et $\pi(z)$ appartiennent à $W$ pour tout $z \in P$ et tout $u \in[0,1]$. L'existence d'un tel $P$ résulte de la continuité des fonctions $\eta, \zeta^{\prime}$ et $H$ et de la condition (ii) du lemme 4.

Si $x \in r_{1}^{-1}(\stackrel{\circ}{s}) \backslash U_{2}$, nous avons $r(\xi(x))=r_{1}(x)$. Quand $x$ tend vers $|\partial s|$, $r_{1} \circ \eta(x)$ et $\pi(x)$ tendent vers $r_{1}(x)$, donc, d'après (i) et la condition (i) $\mathrm{du}$ lemme 4 , si $\varepsilon^{\prime}$ est assez petit, nous avons $d\left((1-u) r \circ H\left(\zeta^{\prime} \circ \eta(x), t\right)+\right.$ $\left.u \pi(x), r_{1}(x)\right)<\varepsilon$ quels que soient $u$ et $t$ si $x$ est assez proche de $|\partial s|$. La condition (i) peut alors être satisfaite en prenant $U_{2}$ assez petit.

Si $x \in \stackrel{\circ}{\sigma}$, alors $\zeta(x)=(1-\mu(x)) \pi(x)+\mu(x) b_{s}=r_{1}(x)=x$ si $x \neq b_{s}$, et $\zeta\left(b_{s}\right)=\xi\left(b_{s}\right)=b_{s}$, d'où (ii).

Soit $x \in r_{1}^{-1}(\stackrel{\circ}{s})$, et soient $\left(b_{\sigma_{0}}, m_{0}\right), \ldots,\left(b_{\sigma_{p}}, m_{p}\right)$, où $\sigma_{0} \leq \ldots \leq \sigma_{p}$ et $m_{0}<\ldots<m_{p}$, les éléments de $V$ sur lesquels $x$ a une coordonnée barycentrique non nulle. Nécessairement, $\left(b_{\sigma_{p}}, m_{p}\right)$ appartient à $S$. Si $x \notin$ $\left|M^{k}\left(K^{(1)}, n\right)\right|$, alors $m_{p}>k$; comme $\xi(x)$, donc aussi $\zeta(x)$, a une coordonnée barycentrique non nulle sur $\left(b_{s}, \max \left(d, m_{p}\right)\right), \zeta(x)$ n'appartient pas à $\left|M^{k}(K, n)\right|$, d'où (iii). Si $\sigma$ est un simplexe de $K^{(1)}$ tel que $|\sigma|$ contienne $r_{1}(x)$, alors $|\sigma|$ contient $\left|\sigma_{p}\right|$ et les points $b_{s}, \pi(x)$ et $r_{1}(\eta(x))$ lorsque ces 
derniers sont définis; puisque $\zeta^{\prime}$ vérifie (iv), si $x \in U_{2}$, alors $|\sigma|$ contient aussi $r\left(\zeta^{\prime} \circ \eta(x)\right)=r \circ H\left(\zeta^{\prime} \circ \eta(x), \gamma(x)\right)$. La condition (iv) en résulte. Reprenant le raisonnement fait pour montrer que $\zeta$ est bien définie, nous constatons que les sommets de $M(K, n)$ sur lesquels $\zeta(x)$ a une coordonnée barycentrique non nulle soit sont de la forme $\left(b_{s}, \max \left(d, m_{j}\right)\right)$, soit appartiennent à $K^{(1)}$, soit sont des sommets du simplexe $\left|\sigma^{\prime}\right|$ de $|M(K, n)|$ contenant $H\left(\zeta^{\prime} \circ \eta(x), \gamma(x)\right)$. Il résulte des constructions de $\eta$ et $H$ et du fait que $\zeta^{\prime}$ vérifie (vi) que les sommets de $\sigma^{\prime}$ sont de la forme $\left(b_{\tau}, m\right)$ où $m=\operatorname{dim} \tau$ ou bien $m=m_{j}$ pour un $j<p$. La condition (vi) est donc aussi vérifiée.

5. Démonstration du lemme 3. Dans tout ce qui suit, si $\mathcal{V}=\left\{V_{\alpha} \mid\right.$ $\alpha \in A\}$ est un recouvrement ouvert de $X$ de nerf $K$, nous supposons $V_{\alpha} \neq \emptyset$ pour tout $\alpha$, ce qui nous permet d'identifier les éléments de $A$ aux sommets de $K$. Une application canonique de $X$ dans $|K|$ est une fonction continue telle que $\psi^{-1}(\operatorname{St}(\alpha, K)) \subset V_{\alpha}$ pour tout $\alpha$. Si $\mathcal{W}=\left\{W_{\beta} \mid \beta \in B\right\}$ est un recouvrement ouvert plus fin que $\mathcal{V}$ et si $L$ est le nerf de $\mathcal{W}$, une projection de $L$ dans $K$ est une application simpliciale $q$ telle que $W_{\beta} \subset U_{q(\beta)}$ pour tout $\beta \in B$.

Fixons une distance $d$ définissant la topologie de $X$. Prenons une suite de recouvrements ouverts finis $\mathcal{V}_{n}=\left\{V_{\alpha} \mid \alpha \in A_{n}\right\}$ de $X$ de façon que, notant $K_{n}$ le nerf de $\mathcal{V}_{n}$, les conditions suivantes soient vérifiées :

$\mathcal{V}_{n+1}$ est plus fin que $\mathcal{V}_{n}$ $\operatorname{dim} K_{n} \leq n$,

toute application canonique de $X$ dans $\left|K_{n}\right|$ est surjective, $\lim _{n \rightarrow \infty} \sup \left\{\operatorname{diam} V_{\alpha} \mid \alpha \in A_{n}\right\}=0$.

Une telle suite peut se construire comme suit : posons $n_{0}=0$ et $\mathcal{V}_{0}=$ $\{X\}$. Supposons que, pour un entier $n_{k}, \mathcal{V}_{n}$ soit construit pour $n \leq n_{k}$ et que diam $V<1 / k$ pour tout $V \in \mathcal{V}_{n_{k}}$ si $k \geq 1$. Soit $\mathcal{V}^{\prime}=\left\{V_{\alpha}^{\prime} \mid \alpha \in A^{\prime}\right\}$ un recouvrement ouvert de $X$ plus fin que $\mathcal{V}_{n_{k}}$ et tel que diam $V_{\alpha}^{\prime}<1 /(k+1)$ pour tout $\alpha \in A^{\prime}$. Soit $K^{\prime}$ le nerf de $\mathcal{V}^{\prime}$. S'il existe une application canonique non surjective $\psi$ de $X$ dans $\left|K^{\prime}\right|$, alors il y a un simplexe maximal $\sigma$ de $K^{\prime}$ et un point $x \in|\sigma| \backslash \psi(X)$. Soit $q$ une rétraction de $\left|K^{\prime}\right| \backslash\{x\}$ sur $\left|K^{\prime}\right| \backslash \stackrel{\circ}{\sigma}$. Posant $\psi^{\prime}=q \circ \psi$, nous avons $\psi^{\prime-1}\left(\operatorname{St}\left(\alpha, K^{\prime}\right)\right) \subset V_{\alpha}^{\prime}$ pour tout $\alpha$, et $\mathcal{V}^{\prime \prime}=\left\{\psi^{\prime-1}\left(\operatorname{St}\left(\alpha, K^{\prime}\right)\right) \mid \alpha \in A^{\prime}\right\}$ est un recouvrement ouvert de $X$ plus fin que $\mathcal{V}^{\prime}$ dont le nerf est un sous-complexe propre de $K^{\prime}$. Répétant cette opération un nombre fini de fois, nous arrivons à un recouvrement ouvert $\mathcal{V}$, plus fin que $\mathcal{V}^{\prime}$, dont le nerf $K$ est un sous-complexe de $K^{\prime}$ et tel que (3) soit vérifié par $\mathcal{V}$. Alors $\mathcal{V}$ est plus fin que $\mathcal{V}_{n_{k}}$ et tout élément de $\mathcal{V}$ a un diamètre $<1 /(k+1)$. Soit $n_{k+1}=\max \left(n_{k}+1\right.$, dim $\left.K\right)$. Posons $\mathcal{V}_{n}=\mathcal{V}_{n_{k}}$ pour $n_{k} \leq n<n_{k+1}$ et $\mathcal{V}_{n_{k+1}}=\mathcal{V}$. La suite ainsi construite vérifie les conditions (1)-(4). 
Pour $n \geq 1$, soit $q_{n}$ une projection de $K_{n+1}$ dans $K_{n}$. Si $\psi$ est une application canonique de $X$ dans $\left|K_{n+1}\right|$, alors $q_{n} \circ \psi$ est une application canonique de $X$ dans $\left|K_{n}\right|$, donc (3) entraîne que $q_{n}$ est surjective.

À toute suite $\left\{\mathcal{V}_{n}\right\}$ de recouvrements ouverts vérifiant les conditions (1), (3) et (4) on peut facilement associer un compact $Z$ et une fonction continue $\varphi$ de $Z$ dans $X$ vérifiant la condition (ii) du lemme 3. Soit $Z$ la limite de la suite projective $\left(\left|K_{n}\right|, q_{n, m}\right)$ où $q_{n, m}=q_{n} \circ \ldots \circ q_{m-1}$ pour $n<m$. Puisque les $q_{n}$ sont surjectives, la projection naturelle $\pi_{n}$ de $Z$ sur $\left|K_{n}\right|$ l'est aussi. Pour $z \in Z$ et $n \geq 1$, soit $\sigma_{n}(z)$ le plus petit simplexe de $K_{n}$ tel que $\left|\sigma_{n}(z)\right|$ contienne $\pi_{n}(z)$, et soit $F_{n}(z)=\bigcap_{\alpha \in \sigma_{n}(z)} \bar{V}_{\alpha}$. La suite $\left\{F_{n}(z)\right\}$ converge vers un point $\varphi(z)$ de $X$. La fonction $\varphi$ ainsi définie est continue et la condition (ii) résulte facilement de l'existence d'applications canoniques $\psi_{n}: X \rightarrow\left|K_{n}\right|$. En effet, soient $N$ un complexe dénombrable localement fini et $\xi:|N| \rightarrow X$ une fonction continue. Pour tout sommet $v$ de $N$, la surjectivité de $\pi_{n}$ entraîne l'existence d'un point $\eta(v)$ de $Z$ tel que $\pi_{n}(\eta(v))=\psi_{n}(\xi(v))$. Prolongeant $\eta$ linéairement sur chaque simplexe, nous obtenons une fonction continue de $|N|$ dans $\left(P(Z), \tau^{\prime}\right)$. Si $N$ est fini, $\eta$ est le relèvement approximatif cherché pourvu que $n$ soit assez grand et la triangulation de $N$ assez fine. Si $N$ est infini, écrivons-le comme réunion d'une suite croissante de sous-complexes finis, construisons des relèvements approximatifs sur ces sous-complexes et recollons linéairement ces relèvements. Malheureusement, le compact $Z$ obtenu par ce procédé simple ne vérifie pas la condition (i) du lemme 3 car il contient en général des copies du cube de Hilbert. Pour éliminer ce problème, il faut diminuer les images réciproques $q_{n}^{-1}(y)$ des points par les applications $q_{n}$, ce que permet la construction géométrique $M(K, n)$ du paragraphe 4 . Nous obtiendrons $Z$ comme limite d'une suite projective $\left(Z_{n}, \pi_{n}^{m}\right)$ où $Z_{n}$ contient $\left|K_{n}\right|$. La fonction $\varphi$ sera construite de façon analogue à celle indiquée plus haut et, pour pouvoir en vérifier la condition (ii) par le raisonnement esquissé ci-dessus, il suffira de s'assurer que $\pi_{n}(Z)$ contient $\left|K_{n}\right|$.

Les compacts $Z_{n}$ seront supposés munis de distances $d_{n}$ majorées par un, et $Z$ sera muni de la distance $d^{\prime}\left(\left(x_{n}\right),\left(y_{n}\right)\right)=\sum_{n=1}^{\infty} 2^{-n} d_{n}\left(x_{n}, y_{n}\right)$. Nous construirons une suite $k_{1}<k_{2}<\ldots$ d'entiers, et nous poserons $M_{n}=$ $M\left(K_{n}^{\left(k_{n}\right)}, n\right)$ et $r_{n}=r\left(K_{n}^{\left(k_{n}\right)}, n\right)$. Pour tout simplexe $\sigma$ de $K_{n}^{\left(k_{n}\right)}$, soit $D_{\sigma}=$ $r_{n}^{-1}(|\sigma|)$ et soit $C_{\sigma}$ une copie du produit $D_{\sigma} \times[0,1]$; nous notons $[\sigma, x, t]$ $\left(x \in D_{\sigma}, t \in[0,1]\right)$ le point générique de $C_{\sigma}$. Nous identifions naturellement $D_{\sigma}$ à $D_{\sigma} \times\{0\} \subset C_{\sigma}$ et supposons que $C_{\sigma} \cap C_{\sigma^{\prime}}=D_{\sigma} \cap D_{\sigma^{\prime}}$ si $\sigma \neq \sigma^{\prime}$. Posons $Z_{n}=\left|M_{n}\right| \cup \bigcup\left\{C_{\sigma} \mid \sigma \in K_{n}^{\left(k_{n}\right)}\right\}$. Soit $p_{n}$ la rétraction de $Z_{n}$ sur $\left|M_{n}\right|$ définie par $p_{n}([\sigma, s, t])=x$ pour $[\sigma, x, t] \in C_{\sigma}$, et soit $\varrho_{n}=r_{n} \circ p_{n}: Z_{n} \rightarrow\left|K_{n}\right|$. Soit $\nu_{n}^{\prime}=\nu\left(K_{n}^{\left(k_{n}\right)}, n\right) \circ p_{n}: Z_{n} \rightarrow[0,1]$. Définissons une fonction continue $\gamma_{n}: Z_{n} \rightarrow[0,1]$ par $\gamma_{n}(x)=0$ si $x \in\left|M_{n}\right|$ et $\gamma_{n}([\sigma, x, t])=t$. Pour $0 \leq k \leq n$, soit $M_{n}^{k}=M^{k}\left(K_{n}^{\left(k_{n}\right)}, n\right)$. Pour $\sigma \in K_{n}^{\left(k_{n}\right)}$, soit $E_{\sigma}^{n}=C_{\sigma} \backslash\left|M_{n}^{n-1}\right|$. 
Les ensembles $E_{\sigma}^{n}, \sigma \in K_{n}^{\left(k_{n}\right)}$, sont ouverts et deux à deux disjoints ; soit $E^{n}=Z_{n} \backslash\left|M_{n}^{n-1}\right|$ leur réunion.

Posons $k_{1}=0$. Supposons que, pour un $n \geq 1, k_{n}$ et les $\pi_{m}^{n}$ soient définis. Pour un sous-ensemble $F$ de $\left|K_{n}\right|$ et $\varepsilon>0$, posons $B(F, \varepsilon)=\{x \in$ $\left.\left|K_{n}\right| \mid d_{n}(x, F)<\varepsilon\right\}$. Remarquons que, pour tout sous-ensemble $F$ de $\left|K_{n}\right|$, nous avons $\varrho_{n}^{-1}(F) \cap \nu_{n}^{-1}(0) \cap \gamma_{n}^{-1}(0)=F$. Puisque $\sup \left\{\operatorname{diam}|s| \mid s \in K_{n}^{(k)}\right\}$ tend vers zéro quand $k$ tend vers l'infini, nous pouvons trouver un entier $k_{n+1}>k_{n}$ et un nombre $0<\varepsilon_{n}<1$ tels que

$$
\operatorname{diam} \pi_{m}^{n}\left(\varrho_{n}^{-1}\left(B\left(|\sigma|, \varepsilon_{n}\right)\right) \cap \nu_{n}^{-1}\left(\left[0, \varepsilon_{n}\right]\right) \cap \gamma_{n}^{-1}\left(\left[0, \varepsilon_{n}\right]\right)\right) \leq 2^{-n} \text { pour tout }
$$
$m \leq n$ et tout simplexe $\sigma$ de $K_{n}^{\left(k_{n+1}\right)}$.

Soit $\widehat{r}_{n}=r\left(K_{n}^{\left(k_{n+1}\right)}, n\right): M\left(K_{n}^{\left(k_{n+1}\right)}, n\right) \rightarrow K_{n}^{\left(k_{n+1}+1\right)}$. Pour tout simplexe $\sigma$ de $K_{n+1}^{\left(k_{n+1}\right)}$, soit $\sigma^{\prime}=q_{n}^{\left(k_{n+1}\right)}(\sigma) \in K_{n}^{\left(k_{n+1}\right)}$. Nous définissons une application simpliciale $\mu_{n}$ de $M_{n+1}^{n}$ dans $M\left(K_{n}^{\left(k_{n+1}\right)}, n\right)$ en envoyant le sommet $\left(b_{\sigma}, m\right)$ de $M_{n+1}^{n} \operatorname{sur}\left(b_{\sigma^{\prime}}, m\right)$. Alors, pour tout simplexe $\sigma$ de $K_{n+1}^{\left(k_{n+1}\right)}$, $\mu_{n}\left(r_{n+1}^{-1}(|\sigma|) \cap\left|M_{n+1}^{n}\right|\right) \subset \widehat{r}_{n}^{-1}\left(\left|q_{n}^{\left(k_{n+1}\right)}(\sigma)\right|\right)$; d'après le lemme 4 , ce dernier ensemble est contractile, et, puisque les ensembles $E_{\sigma}^{n+1}, \sigma \in K_{n+1}^{\left(k_{n+1}\right)}$, sont deux à deux disjoints, nous pouvons prolonger $\mu_{n}$ en une fonction continue, encore notée $\mu_{n}$, de $Z_{n+1}$ dans $\left|M\left(K_{n}^{\left(k_{n+1}\right)}, n\right)\right|$ vérifiant

$$
\mu_{n}\left(C_{\sigma}\right) \subset \widehat{r}_{n}^{-1}\left(\left|q_{n}^{\left(k_{n+1}\right)}(\sigma)\right|\right) \quad \text { pour tout simplexe } \sigma \in K_{n+1}^{\left(k_{n+1}\right)} .
$$

Soit $\zeta_{n}:\left|M\left(K_{n}^{\left(k_{n+1}\right)}, n\right)\right| \rightarrow\left|M_{n}\right|$ la fonction fournie par le lemme 6, appliqué à $K=K_{n}^{\left(k_{n}\right)}, l=k_{n+1}-k_{n}$ et $\varepsilon=\varepsilon_{n}$, et soit $\theta_{n}=\zeta_{n} \circ \mu_{n}$ : $Z_{n+1} \rightarrow\left|M_{n}\right|$. Pour un simplexe $\sigma$ de $K_{n+1}^{\left(k_{n+1}\right)}$, soit $\widehat{\sigma}$ le plus petit simplexe de $K_{n}^{\left(k_{n}\right)}$ tel que $\left|q_{n}^{\left(k_{n+1}\right)}(\sigma)\right| \subset|\widehat{\sigma}|$. D'après (6) et la condition (iv) du lemme $6, \theta_{n}\left(C_{\sigma}\right) \subset r_{n}^{-1}(|\widehat{\sigma}|)=D_{\widehat{\sigma}}$, donc, prenant une fonction continue $\beta_{n+1}: Z_{n+1} \rightarrow\left[0, \varepsilon_{n}\right]$ telle que $\beta_{n+1}^{-1}(0)=\left|M_{n+1}^{n}\right|$, nous pouvons définir $\pi_{n}^{n+1}: Z_{n+1} \rightarrow Z_{n}$ par

$$
\pi_{n}^{n+1}(x)= \begin{cases}\theta_{n}(x) & \text { si } x \in\left|M_{n+1}^{n}\right|, \\ {\left[\widehat{\sigma}, \theta_{n}(x), \beta_{n+1}(x)\right]} & \text { si } x \in \overline{E_{\sigma}^{n+1}}, \sigma \in K_{n+1}^{\left(k_{n+1}\right)} .\end{cases}
$$

Pour $m<n$, posons $\pi_{m}^{n+1}=\pi_{m}^{n} \circ \pi_{n}^{n+1}$. Remarquons que

$$
\begin{aligned}
& \left(\pi_{n}^{n+1}\right)^{-1}\left(\left|M_{n}^{k}\right|\right) \subset\left|M_{n+1}^{k}\right| \text { pour tout } k \leq n-1, \\
& \operatorname{diam} \pi_{m}^{n+1}\left(E_{\sigma}^{n+1}\right) \leq 2^{-n} \text { pour tout } m \leq n \text { et pour tout } \sigma \in K_{n+1}^{\left(k_{n+1}\right)},
\end{aligned}
$$
$\left|K_{n}\right| \subset \pi_{n}^{n+1}\left(\left|K_{n+1}\right|\right)$.

Preuve de (7) : Soit $x \notin\left|M_{n+1}^{k}\right|$. Si $x \notin\left|M_{n+1}^{n}\right|$, alors $\beta_{n+1}(x) \neq 0$, et il existe $\widehat{\sigma}$ tel que $\pi_{n}^{n+1}(x) \in C_{\widehat{\sigma}} \backslash\left|M_{n}\right|$. Si $x \in\left|M_{n+1}^{n}\right|$, alors, d'après la définition de $\mu_{n}, \mu_{n}(x) \notin\left|M^{k}\left(K_{n}^{\left(k_{n+1}\right)}, n\right)\right|$, et la condition (iii) du lemme 6 entraîne que $\pi_{n}^{n+1}(x)=\zeta_{n} \circ \mu_{n}(x) \notin\left|M_{n}^{k}\right|$. 
Le choix de $\beta_{n+1},(7)$ et les conditions (i) et (v) du lemme 6 entraînent que, pour tout simplexe $\sigma$ de $K_{n+1}^{\left(k_{n+1}\right)}$ et $\sigma^{\prime}=q_{n}^{\left(k_{n+1}\right)}(\sigma)$, nous avons

$$
\pi_{n}^{(n+1)}\left(E_{\sigma}^{n+1}\right) \subset \varrho_{n}^{-1}\left(B\left(\left|\sigma^{\prime}\right|, \varepsilon_{n}\right)\right) \cap \nu_{n}^{\prime-1}\left(\left[0, \varepsilon_{n}\right]\right) \cap \gamma_{n}^{-1}\left(\left[0, \varepsilon_{n}\right]\right),
$$

donc (8) résulte de (5).

Puisque $q_{n}$ est surjective, pour tout simplexe $s$ de $K_{n}$, il y a un simplexe $\sigma$ de $K_{n+1}$, de même dimension que $s$, tel que $q_{n}(\sigma)=s$. Alors, $q_{n} \mid \sigma$ est un isomorphisme de $\sigma$ sur $s$, et $q_{n}^{\left(k_{n+1}+1\right)}$ un isomorphisme simplicial de $\sigma^{\left(k_{n+1}+1\right)} \operatorname{sur} s^{\left(k_{n+1}+1\right)}$. En se reportant à la façon dont $K_{n+1}^{\left(k_{n+1}+1\right)}$ est identifié à un sous-complexe de $M_{n+1}$ et à la définition de $\mu_{n}$, on constate que $\sigma^{\left(k_{n+1}+1\right)} \subset M_{n+1}^{n}\left(\operatorname{car} \operatorname{dim} \sigma \leq \operatorname{dim} K_{n} \leq n\right)$ et que $\mu_{n} \mid \sigma^{\left(k_{n+1}+1\right)}=$ $q_{n}^{\left(k_{n+1}+1\right)} \mid \sigma^{\left(k_{n+1}+1\right)}$. En utilisant la condition (ii) du lemme 6, nous obtenons $\pi_{n}^{n+1}(|\sigma|)=\zeta_{n} \circ \mu_{n}(|\sigma|)=\mu_{n}(|\sigma|)=q_{n}^{\left(k_{n+1}+1\right)}(|\sigma|)=|s|$. Puisque $s$ est arbitraire, (9) en résulte.

Pour $n \geq 1$, soit $\pi_{n}$ la projection canonique de $Z$ dans $Z_{n}$. Posons $Y_{0}=\bigcap_{n=1}^{\infty} \pi_{n}^{-1}\left(E^{n}\right)$ et $Y_{n}=\pi_{n}^{-1}\left(\left|M_{n}^{n-1}\right|\right)$ pour $n \geq 1$. Puisque $E^{n}=$ $Z_{n} \backslash\left|M_{n}^{n-1}\right|$, nous avons $Z=\bigcup_{n=0}^{\infty} Y_{n}$, donc, pour prouver que $Z$ est de dimension dénombrable, il suffit de montrer que les $Y_{n}$ sont de dimension finie. Pour $n \geq 1, Y_{n}=\lim \left(\pi_{m}\left(Y_{n}\right), \pi_{m}^{l} \mid \pi_{l}\left(Y_{n}\right)\right)$. Il résulte de (7) que $\pi_{m}\left(Y_{n}\right) \subset\left|M_{m}^{n-1}\right|$ pour tout $m \geq n$; comme $\operatorname{dim}\left|M_{m}^{n-1}\right|=n-1, Y_{n}$ est donc limite d'une suite projective de compacts de dimensions $\leq n-1$, donc est de dimension $\leq n-1$. Pour tout $n \geq 1$, la famille d'ouverts disjoints $\left\{\pi_{n}^{-1}\left(E_{\sigma}^{n}\right) \mid \sigma \in K_{n}^{\left(k_{n}\right)}\right\}$ recouvre $Y_{0}$, et il résulte de (8) que

$$
\operatorname{diam} \pi_{n}^{-1}\left(E_{\sigma}^{n}\right) \leq\left(\sum_{m=1}^{n-1} 2^{-m}\right) 2^{-(n-1)}+\sum_{m=n}^{\infty} 2^{-m}<2^{-(n-2)},
$$

ce qui entraîne que $\operatorname{dim} Y_{0}=0$.

Pour tout $n \geq 1$ et tout point $z$ de $Z$ soient $\sigma_{n}(z)$ le plus petit simplexe de $K_{n}^{\left(k_{n}\right)}$ tel que $\pi_{n}(z) \in C_{\sigma_{n}(z)}, s_{n}(z)$ le plus petit simplexe de $K_{n}$ tel que $\left|s_{n}(z)\right|$ contienne $\left|\sigma_{n}(z)\right|$, et $F_{n}(z)=\bigcap_{\alpha \in s_{n}(z)} \bar{V}_{\alpha} . F_{n}(z)$ est un fermé de $X$ vérifiant

$$
\operatorname{diam} F_{n}(z) \leq \Delta_{n}=\max \left\{\operatorname{diam} \bar{V}_{\alpha} \mid \alpha \in A_{n}\right\} .
$$

Pour $n<m$, soit $q_{n, m}=q_{n} \circ \ldots \circ q_{m-1}$; c'est une projection de $K_{m}$ dans $K_{n}$. Nous avons $s_{n}(z) \subset q_{n, m}\left(s_{m}(z)\right)$. En effet, il suffit, par récurrence, de le vérifier quand $m=n+1$. Soit $\widehat{\sigma}$ le plus petit simplexe de $K_{n}^{\left(k_{n}\right)}$ tel que $q_{n}^{\left(k_{n+1}\right)}\left(\left|\sigma_{n+1}(z)\right|\right) \subset|\widehat{\sigma}|$. Par construction de $\pi_{n}^{n+1}, \pi_{n}(z)=$ $\pi_{n}^{n+1}\left(\pi_{n+1}(z)\right)$ est contenu dans $C_{\widehat{\sigma}}$, donc $\sigma_{n}(z) \subset \widehat{\sigma}$. Mais $q_{n}\left(\left|s_{n+1}(z)\right|\right)$ contient $q_{n}^{\left(k_{n+1}\right)}\left(\left|\sigma_{n+1}(z)\right|\right)$, donc aussi $|\widehat{\sigma}|$ et, a fortiori, $\left|\sigma_{n}(z)\right|$, d'où l'inclusion $s_{n}(z) \subset q_{n}\left(s_{n+1}(z)\right)$. Puisque $q_{n, m}$ est une projection, nous avons $F_{n}(z) \cup$ $F_{m}(z) \subset \bigcup\left\{\bar{V}_{\alpha} \mid \alpha \in q_{n, m}\left(s_{m}(z)\right)\right\}$; comme $q_{n, m}\left(s_{m}(z)\right)$ est un simplexe de 
$K_{n}$, le diamètre de ce dernier ensemble est $\leq 2 \Delta_{n}$, d'où (11) $\quad d(x, y) \leq 2 \Delta_{n} \quad$ quels que soient $n \leq m, x \in F_{n}(z)$ et $y \in F_{m}(z)$.

Il résulte de (4), (10) et (11) que la suite de fermés $\left\{F_{n}(z)\right\}$ converge vers un point $\varphi(z)$ de $X$. Si $\alpha \in A_{n}$ est tel que $\varrho_{n}\left(\pi_{n}(z)\right) \in \operatorname{St}\left(\alpha, K_{n}\right)$, alors $\alpha \in s_{n}(z)$, donc $F_{n}(z) \subset \bar{V}_{\alpha}$, et (11) entraîne

$$
\begin{array}{r}
d\left(\varphi(z), \bar{V}_{\alpha}\right) \leq 2 \Delta_{n} \quad \text { pour tout } z \in Z \text { et tout } \alpha \in A_{n} \\
\text { tels que } \varrho_{n}\left(\pi_{n}(z)\right) \in \operatorname{St}\left(\alpha, K_{n}\right) .
\end{array}
$$

Pour tout $n$, les ensembles $\left(\varrho_{n} \circ \pi_{n}\right)^{-1}\left(\operatorname{St}\left(\alpha, K_{n}\right)\right), \alpha \in A_{n}$, forment un recouvrement ouvert de $Z$. Si $z, z^{\prime}$ appartiennent à $\left(\varrho_{n} \circ \pi_{n}\right)^{-1}\left(\operatorname{St}\left(\alpha, K_{n}\right)\right)$, il résulte de (12) que

$$
d\left(\varphi(z), \varphi\left(z^{\prime}\right)\right) \leq d\left(\varphi(z), \bar{V}_{\alpha}\right)+\operatorname{diam} \bar{V}_{\alpha}+d\left(\varphi\left(z^{\prime}\right), \bar{V}_{\alpha}\right) \leq 5 \Delta_{n},
$$

d'où la continuité de $\varphi$ puisque, d'après $(4), \lim _{n \rightarrow \infty} \Delta_{n}=0$.

Soient $\tau \in \mathcal{T}(X)$ et $\tau^{\prime} \in \mathcal{T}(Z)$ telles que $\widehat{\varphi}:\left(P(Z), \tau^{\prime}\right) \rightarrow(P(X), \tau)$ soit continue. Nous prolongeons la distance $d$ (resp. $\left.d^{\prime}\right)$ de $X$ (resp. $Z$ ) en une distance, encore notée $d\left(\right.$ resp. $\left.d^{\prime}\right)$ sur $P(X)$ (resp. $P(Z)$ ) définissant la topologie $\tau$ (resp. $\tau^{\prime}$ ).

Soient $\mathcal{U}$ un recouvrement $\tau$-ouvert de $P(X), N$ un complexe simplicial dénombrable localement fini et $\xi:|N| \rightarrow X$ une fonction continue. Quitte à subdiviser $N$, nous pouvons l'écrire $N=\bigcup_{i=1}^{\infty} N_{i}$ où, pour tout $i, N_{i}$ est un complexe fini de dimension $\leq i$ tel que $\left|N_{i}\right| \subset \operatorname{Int}\left|N_{i+1}\right|$. La compacité de $X$ nous permet de trouver un $e>0$ vérifiant

Tout sous-ensemble $G$ de $P(X)$ de diamètre $<e$ tel que $G \cap X \neq \emptyset$ est contenu dans un élément de $\mathcal{U}$.

Pour $i \geq 1$, soit $O_{i}=\left\{z \in P(Z) \mid d^{\prime}\left(z, P_{2}(Z)\right)<1 / i\right\}$. Pour un sousensemble $H$ de $P(Z)$, nous notons $\operatorname{conv}(H)$ son enveloppe convexe. Pour tout $i \geq 1$, nous pouvons trouver un nombre $\delta_{i}>0$ vérifiant les deux conditions suivantes :

Si $H_{1}$ et $H_{2}$ sont deux sous-ensembles de $Z$ contenant chacun au plus $i+1$ points, et si diam $H_{1}<\delta_{i}$ et $\operatorname{diam} H_{2}<\delta_{i}$, alors $\operatorname{conv}\left(H_{1} \cup H_{2}\right) \subset O_{i}$.

Si $H$ est un sous-ensemble de $Z$ contenant au plus $2 i+2$ points et si $\operatorname{diam}(\varphi(H))<\delta_{i}, \operatorname{alors} \operatorname{diam}(\widehat{\varphi}(\operatorname{conv}(H)))<e / 2$.

Nous pouvons évidemment supposer $e>\delta_{i} \geq \delta_{i+1}$ pour tout $i$. Puisque $\left\{\Delta_{n}\right\}$ tend vers zéro, nous pouvons inductivement construire une suite $n_{1} \leq$ $n_{2} \leq \ldots$ d'entiers de façon que $\Delta_{n_{1}} \geq \Delta_{n_{2}} \geq \ldots$, que $2^{-n_{i}}<\delta_{i} / 2$ et que $\Delta_{n_{i}}<\delta_{i} / 6$ pour tout $i$. Soit $\psi_{i}: X \rightarrow\left|K_{n_{i}}\right|$ une application canonique. Choisissons inductivement une suite $l_{1} \leq l_{2} \leq \ldots$ d'entiers de façon que, pour tout simplexe $s$ de $N_{i}^{\left(l_{i}\right)}, \operatorname{diam}\left(\pi_{m}^{n_{i}}\left(\psi_{i} \circ \xi(|s|)\right)\right)<2^{-n_{i}}$ pour tout 
$m \leq n_{i}$ et qu'il existe $\alpha \in A_{n_{i}}$ tel que $\psi_{i}(\xi(|s|)) \subset \operatorname{St}\left(\alpha, K_{n_{i}}\right)$. Cela est possible car $\sup \left\{\operatorname{diam}|s| \mid s \in N_{i}^{(l)}\right\}$ tend vers zéro quand $l$ tend vers l'infini. Il résulte de (9) que $\pi_{n_{i}}(Z)$ contient $\left|K_{n_{i}}\right|$. Nous pouvons donc trouver, pour tout sommet $v$ de $N_{i}^{\left(l_{i}\right)}$, un point $\eta_{i}(v)$ de $Z$ tel que $\pi_{n_{i}}\left(\eta_{i}(v)\right)=\psi_{i}(\xi(v))$. Prolongeant linéairement sur chaque simplexe de $N_{i}^{\left(l_{i}\right)}$, nous obtenons une fonction continue $\eta_{i}:\left|N_{i}\right| \rightarrow\left(P(Z), \tau^{\prime}\right)$. Pour $i \geq 1$, prenons une fonction continue $\omega_{i}:|N| \rightarrow[0,1]$ telle que $\omega_{i}(x)=0$ si $x \in\left|N_{i-1}\right|\left(\left|N_{0}\right|=\emptyset\right)$ et $\omega_{i}(x)=1$ si $x \notin \operatorname{Int}\left|N_{i}\right|$. Définissons une fonction continue $\eta:|N| \rightarrow$ $\left(P(Z), \tau^{\prime}\right)$ par

$\eta(x)=\left(1-\omega_{i}(x)\right) \eta_{i}(x)+\omega_{i}(x) \eta_{i+1}(x) \quad$ pour $x \in\left|N_{i}\right| \backslash \operatorname{Int}\left|N_{i-1}\right|, i \geq 1$.

Soit $x \in\left|N_{i}\right| \backslash \operatorname{Int}\left|N_{i-1}\right|$, et soient $s_{i}$ et $s_{i+1}$ des simplexes de $N_{i}^{\left(l_{i}\right)}$ et $N_{i}^{\left(l_{i+1}\right)}$ respectivement tels que $\left|s_{i}\right|$ et $\left|s_{i+1}\right|$ contiennent $x$. Soient $H_{i}=$ $\left\{\eta_{i}(v) \mid v \in s_{i}\right\}$ et $H_{i+1}=\left\{\eta_{i+1}(v) \mid v \in s_{i+1}\right\}$. Puisque $\operatorname{dim} N_{i} \leq i$, chacun des ensembles $H_{i}$ et $H_{i+1}$ contient au plus $i+1$ éléments. Si $v, v^{\prime}$ sont des sommets de $s_{i}$, nous avons

$$
\begin{aligned}
d^{\prime}\left(\eta_{i}(v), \eta_{i}\left(v^{\prime}\right)\right)= & \sum_{n=1}^{\infty} 2^{-n} d_{n}\left(\pi_{n}\left(\eta_{i}(v)\right), \pi_{n}\left(\eta_{i}\left(v^{\prime}\right)\right)\right) \\
= & \sum_{n=1}^{n_{i}} 2^{-n} d_{n}\left(\pi_{n}^{n_{i}}\left(\psi_{i} \circ \xi(v)\right), \pi_{n}^{n_{i}}\left(\psi_{i} \circ \xi\left(v^{\prime}\right)\right)\right) \\
& +\sum_{n=n_{i}+1}^{\infty} 2^{-n} d_{n}\left(\pi_{n}\left(\eta_{i}(v)\right), \pi_{n}\left(\eta_{i}\left(v^{\prime}\right)\right)\right) \\
< & \left(\sum_{n=1}^{n_{i}} 2^{-n}\right) 2^{-n_{i}}+\sum_{n=n_{i}+1}^{\infty} 2^{-n}<2 \cdot 2^{-n_{i}}<\delta_{i},
\end{aligned}
$$

donc diam $H_{i}<\delta_{i}$. De même, diam $H_{i+1}<\delta_{i+1} \leq \delta_{i}$. D'après (14), $\operatorname{conv}\left(H_{i} \cup H_{i+1}\right) \subset O_{i}$. Puisque $\eta(x)$ appartient à $\operatorname{conv}\left(H_{i} \cup H_{i+1}\right)$, cela montre que $\eta\left(\left|N_{i}\right| \backslash \operatorname{Int}\left|N_{i-1}\right|\right)$ est contenu dans $O_{i}$ et, puisque la suite $\left\{O_{i}\right\}$ est décroissante, il en résulte que $\eta(|N|) \backslash O_{i} \subset \eta\left(\left|N_{i-1}\right|\right)$ pour tout $i$. Puisque $\left|N_{i-1}\right|$ est compact et que $\left\{O_{i}\right\}$ est une base de $\tau^{\prime}$-voisinages du compact $P_{2}(Z)$, cela entraîne que $\eta(|N|) \cup P_{2}(Z)$ est $\tau^{\prime}$-compact.

Soit $\alpha \in A_{n_{i}}$ tel que $\psi_{i} \circ \xi\left(\left|s_{i}\right|\right) \subset \operatorname{St}\left(\alpha, K_{n_{i}}\right)$. Puisque $\psi_{i}$ est une application canonique, $\xi(x)$ appartient à $V_{\alpha}$. Pour tout sommet $v$ de $s_{i}$, nous avons, en utilisant (12),

$$
d\left(\varphi\left(\eta_{i}(v)\right), \xi(x)\right) \leq d\left(\varphi\left(\eta_{i}(v)\right), \bar{V}_{\alpha}\right)+\operatorname{diam} \bar{V}_{\alpha} \leq 3 \Delta_{n_{i}} .
$$

De même, $d\left(\varphi\left(\eta_{i+1}\left(v^{\prime}\right)\right), \xi(x)\right) \leq 3 \Delta_{n_{i+1}} \leq 3 \Delta_{n_{i}}$ pour tout sommet $v^{\prime}$ de $s_{i+1}$. Par suite, si $H=H_{i} \cup H_{i+1}$, alors $\operatorname{diam}(\varphi(H)) \leq 6 \Delta_{n_{i}}<\delta_{i}$. Prenant 
un sommet $v$ de $s_{i}$ et utilisant (15), nous obtenons

$$
\begin{aligned}
d(\xi(x), \widehat{\varphi} \circ \eta(x)) & \leq d\left(\xi(x), \varphi\left(\eta_{i}(v)\right)\right)+d\left(\varphi\left(\eta_{i}(v)\right), \widehat{\varphi} \circ \eta(x)\right) \\
& \leq d\left(\xi(x), \varphi\left(\eta_{i}(v)\right)\right)+\operatorname{diam}(\widehat{\varphi}(\operatorname{conv}(H))) \\
& \leq 3 \Delta_{n_{i}}+e / 2<\delta_{i} / 2+e / 2<e,
\end{aligned}
$$

ce qui, d'après (13), montre que $\xi$ est $\mathcal{U}$-proche de $\widehat{\varphi} \circ \eta$.

\section{Bibliographie}

[1] R. Cauty, Un espace métrique linéaire qui n'est pas un rétracte absolu, Fund. Math. 146 (1994), 85-99.

[2] J. Dugundji and A. Granas, Fixed Point Theory, PWN, Warszawa, 1982.

[3] V. V. Fedorchuk and A. Chigogidze, Rétractes absolus et variétés de dimension infinie, Nauka, Moscou, 1992 (en russe).

[4] J. H. Gresham, A class of infinite-dimensional spaces. Part II: An extension theorem and the theory of retracts, Fund. Math. 106 (1980), 237-245.

[5] S. Ulam (éditeur), The Scottish Book, polycopié, 1957.

[6] M. Zarichnyi, Universal maps of $\sigma$ onto $\Sigma$ and absorbing sets in the classes of absolute borelian and projective finite-dimensional maps, Topology Appl. 67 (1995), 221-230.

Université Paris 6

UFR 920

Boîte courrier 172

4, place Jussieu

75252 Paris Cedex 05, France

E-mail: cauty@math.jussieu.fr

Received 28 December 1998;

in revised form 9 October 2000 and 18 June 2001 